\title{
Low-frequency noise of unipolar nanorectifiers
}

\author{
Claudio Balocco, ${ }^{\text {a) }}$ Shahrir R. Kasjoo, Linqing Q. Zhang, Yasaman Alimi, \\ and Aimin M. Song ${ }^{\text {b) }}$ \\ School of Electrical and Electronic Engineering, University of Manchester, Manchester M13 9PL, \\ United Kingdom
}

(Received 8 July 2011; accepted 22 August 2011; published online 14 September 2011)

\begin{abstract}
Unipolar nanodiodes, also known as self-switching devices, have recently been demonstrated as terahertz detectors at room temperature. Here, we study their low-frequency noise spectra and noise equivalent power and show that both performance parameters are comparable to those reported for state-of-the-art Schottky diodes. The truly planar nanodiode layout enables building structures with thousands of devices connected in parallel, which reduce low-frequency noise without affecting sensitivity. The observed $1 / f$ noise can be described by Hooge's mobility fluctuation theory. (C) 2011 American Institute of Physics. [doi:10.1063/1.3636437]
\end{abstract}

Unipolar nanodiodes, known as self-switching devices (SSDs), ${ }^{1-11}$ have shown promising properties as roomtemperature detectors at microwave ${ }^{2}$ and terahertz $(\mathrm{THz})$ frequencies $^{3-5}$ and more recently have been proposed as $\mathrm{THz}$ emitters. ${ }^{6}$ The SSDs consist of two L-shaped trenches which tailor an asymmetric nanochannel in a semiconductor material [Fig. 1(a)]. The geometrical asymmetry of the nanochannel results in a non-linear, diode-like, current-voltage $(I-V)$ characteristic [Fig. 1(b)]. The SSD has been demonstrated in a variety of materials, including two-dimensional electron gases (2DEGs) in $\mathrm{GaAs}^{3}$ and $\mathrm{InGaAs}^{1,2,4}$ silicon-on-insulator $^{7}$ and both organic ${ }^{8}$ and metal-oxide ${ }^{9}$ thin films. The threshold voltage of the SSD is solely defined by the geometry, without the burden of interface junctions or tunneling barriers, and zero-threshold detectors can be easily fabricated. The truly planar nature of the SSD enables the fabrication of arrays with a large number of SSDs connected in parallel in a single fabrication step, as shown in Fig. 1(a) without the need of interconnection layers. Details of its working principle can be found elsewhere. ${ }^{1,5,12}$ However, the experimental characterization of low-frequency $(\mathrm{kHz})$ noise of ultra-fast SSDs in 2DEGs for THz detection application has been so far largely unexplored, although it is of paramount importance for comparison with other wellestablished devices, such as Schottky diodes ${ }^{13}$ and resonant THz detectors based on the Dyakonov-Shur effect. ${ }^{14}$ Theoretical work on noise in SSDs has only been carried out at frequencies higher than $100 \mathrm{GHz} .^{10,11}$

In this letter, we report on the low-frequency noise characterization of a large SSD array with approximately 2000 devices connected in parallel. Low-frequency noise is a critical parameter for direct $\mathrm{THz}$ detectors, which are typically modulated at $10 \mathrm{kHz}$ or below, ${ }^{13}$ as well as rectifier used as frequency mixer in a heterodyne detection scheme. ${ }^{13}$ Connecting such a large number of devices in parallel aids the reduction of the total resistance, thus reducing thermal noise. Increasing the amount of electrons responsible for the conduction also averages out the impact of $1 / f$ or flicker noise. The principle was tested on an SSD array fabricated within

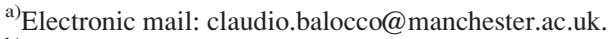

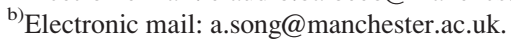

the fingers of an interdigital structure, located in the gaps of a coplanar waveguide as shown in Fig. 2.

The devices were fabricated out of a commercial latticematched InGaAs/InAlAs heterostructure, grown onto a 4-in. InP substrate (purchased from IQE Inc.). The 2DEG, confined in the InGaAs quantum well, was located $25 \mathrm{~nm}$ below the surface. The electron concentration and mobility at room temperature were $1.3 \times 10^{12} \mathrm{~cm}^{-2}$ and $10400 \mathrm{~cm}^{2} / \mathrm{Vs}$, respectively, as determined by Hall measurements. SSD arrays were fabricated by electron-beam lithography (EBL) and wet chemical etch. The SSD channels were approximately $1500 \mathrm{~nm}$ long and $130 \mathrm{~nm}$ wide, whereas the trenches were about $200 \mathrm{~nm}$ wide and $45 \mathrm{~nm}$ deep, as determined by atomic-force micrographs, see Fig. 1(a).

Figure 1(b) shows the current-voltage $(I-V)$ characteristic of the whole array. At relatively high bias, $V \gg k T / q$, where $k$ is the Boltzmann's constant, $T$ is the absolute temperature, and $q$ is the electronic charge; the $I-V$ characteristic is well described by a quadratic approximation. ${ }^{12}$ This model is based on the equations which describe the fieldeffect transistor connected as a diode, as it was found to predict accurately the current. It was also shown that the mobility in the SSD channel did not degrade, albeit the high surface-to-volume ratio. However, at a smaller bias, when the channel is almost completely depleted by the surface states, the current flowing through the SSD is better described by an exponential curve, in analogy to the subthreshold conduction models used for conventional FETs. ${ }^{15}$ In this regime, we can write

$$
I=I_{0}\left[\exp \left(\frac{q V}{n k T}\right)-1\right],
$$

where $I_{0}$ and $n$ are fitting parameters. Fitting the experimental data at room temperature (dashed line in Fig. 1(b)) yields $I_{0}=117 \mu \mathrm{A}$ and $n \sim 28$. The high value of $n$ was ascribed to the parameter spreading of individual SSDs in the array, which also reduced the steepness of the $I-V$ characteristic. The detection sensitivity measured in the range $0-110 \mathrm{GHz}$, approximately $160 \mathrm{~V} / \mathrm{W}$, was reasonably flat and it is not expected to alter significantly at $\mathrm{THz}$ frequencies, as we previously observed. ${ }^{3}$ 

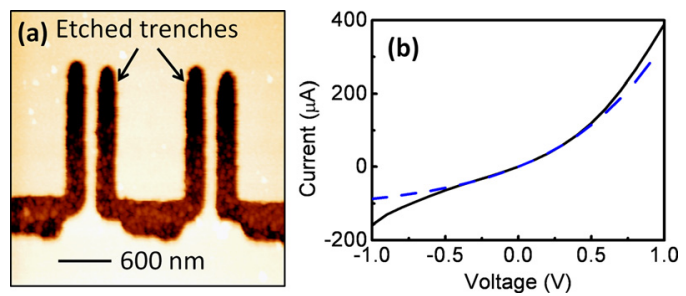

FIG. 1. (Color online) (a) Atomic-force micrograph of two SSDs connected in parallel. The channel length and width are $1500 \mathrm{~nm}$ and $130 \mathrm{~nm}$, respectively. The trench width and depth are $200 \mathrm{~nm}$ and $45 \mathrm{~nm}$, respectively. (b) $I-V$ characteristic of the SSD array measured in dark at room temperature. The dashed line shows the exponential fitting at low biases (see text).

In order to measure the noise power spectra, the SSD array was bonded onto a printed circuit board (PCB), which was then mounted in a metallic box to screen external radiofrequency and $50-\mathrm{Hz}$ line interferences. The voltage fluctuation across the SSD array, $s_{n}(t)$, was first amplified with a custom low-noise amplifier running on battery with a gain of approximately $10^{4}$. The amplified signal was then sampled by a 16-bit analog-to-digital converter (ADC) with a variable rate $\left(S_{\text {rate }}\right)$ up to $125 \mathrm{kS} / \mathrm{s}$ for a time period $t_{0}$. The power spectrum $S_{n}(f)$ was computed using the square of module of the discrete Fourier transform (DFT) $\left[s_{n}(t)\right]$ divided by the square of the amplifier gain. Several time sequences were measured, and their power spectra were averaged to obtain the final noise power spectrum. The control of the ADC as well as the computation and averaging of the power spectra were automated using Labview. The lowest measured frequency depends on the acquisition time $f_{L}=1 / t_{0}$, whereas the highest depends on the sampling rate as $f_{H}=S_{\text {rate }} / 2 \sim 60 \mathrm{kHz}$ following Nyquist's sampling theorem. However, the highest frequency was limited by the amplifier bandwidth $(-3 \mathrm{~dB})$ of approximately $40 \mathrm{kHz}$. The highest frequency was further reduced to $10 \mathrm{kHz}$ to minimize the effect of the amplifier frequency dependence. Two different time windows were used in the reported measurements: $t_{0}=10 \mathrm{~s}$ for the frequency range $0.1 \mathrm{~Hz}-10 \mathrm{~Hz}$ with 100 averaged spectra and $t_{0}=0.1 \mathrm{~s}$ for the frequency range $10 \mathrm{~Hz}-10 \mathrm{kHz}$ with 1000 averaged spectra. The system was calibrated by measuring the thermal noise of several thinfilm resistors in the range $1-100 \mathrm{k} \Omega$. The SSD array was biased by forcing a continuous (dc) current through a $10 \mathrm{M} \Omega$ resistor and a battery-run voltage source. In a small signal analysis, this large resistor is in parallel to the SSD array, which has a much lower resistance, and hence its noise contribution can be neglected.

The noise spectra at different bias currents are shown in Fig. 3. At zero bias, the SSD array had a resistance $R_{0}$ of approximately $6.2 \mathrm{k} \Omega$, estimated from Fig. 1(b), which gen-

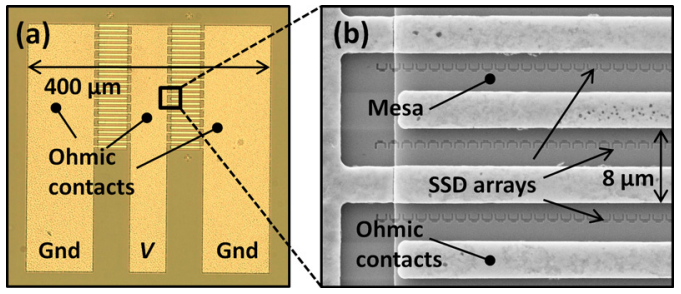

FIG. 2. (Color online) (a) Optical micrograph of the interdigital structure and coplanar waveguide. (b) SEM micrograph showing the detail of the interdigital-structure fingers with SSDs located within.

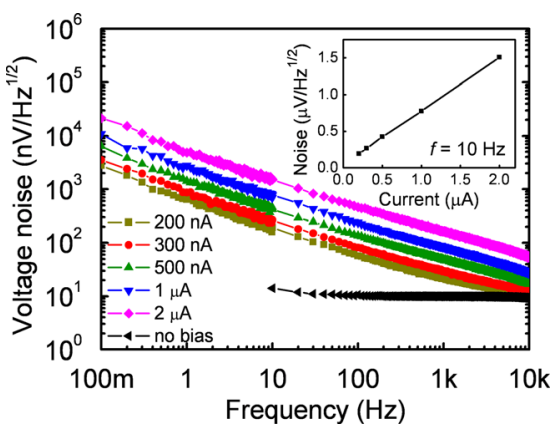

FIG. 3. (Color online) Room-temperature voltage-noise spectra of the SSD array at different biases. The small deviation from a white noise spectrum at low frequencies of the unbiased array is due to residual $1 / f$ noise of the amplifier. The inset shows the linear dependence of the voltage noise (equal to the square root of the noise power) as a function of the current at $10 \mathrm{~Hz}$.

erated a thermal noise ${ }^{16} 4 k T R_{0}=10.2 \mathrm{nV} / \mathrm{Hz}^{1 / 2}$, and agreed well with the measured value of $\sim 10 \mathrm{nV} / \mathrm{Hz}^{1 / 2}$. When a bias was applied, the differential resistance $R$ of the SSD array could be calculated from Eq. (1) as

$$
R=\frac{d V}{d I}=\frac{n k T}{q} \frac{1}{I_{0}\left(I / I_{0}+1\right)}=R_{0} \frac{1}{\left(I / I_{0}+1\right)} .
$$

Since in our measurements $I$ ranged from 0 to $2 \mu \mathrm{A}$ and $I_{0}$ is $177 \mu \mathrm{A}$, we can safely considered a constant resistance $R$ $\sim R_{0}$ with an error smaller than $2 \%$.

When a bias was applied to the SSD array, $1 / f$ (flicker) noise arose in accordance to Hooge's mobility fluctuation theory $^{16,17}$

$$
\frac{S_{I}(f)}{I^{2}} \equiv \frac{S_{V}(f)}{V^{2}} \equiv \frac{S_{V}(f)}{I^{2} R^{2}}=\frac{\alpha_{H}}{N f^{\beta}},
$$

where $S_{I}(f)$ and $S_{V}(f)$ are the current and voltage noise power spectra, respectively, $N$ is the number of carriers in the SSDs' channels responsible for conduction, $\beta$ is a fitting parameter close to 1 , and $\alpha_{H}$ is the Hooge's constant. As expected, the power of the $1 / f$ noise was found proportional to $I^{2}$, see inset in Fig. 3. The number of carrier $N$ can be estimated from the resistance of the SSD array and the 2DEG mobility $\mu_{n}$; in the linear regime, the carrier density $n_{S S D}$ inside the SSD's channels can be written as

$$
n_{S S D}=\frac{L}{W_{e f f} q \mu_{n} M R},
$$

where $L$ is the SSDs' channel length, $W_{\text {eff }}$ is the effective channel width considering the depletion due to surface states, and $M$ is the number of SSDs in the array. The total number of carriers in the whole array is, thus,

$$
N=M n_{S S D} W_{e f f} L=\frac{L^{2}}{q \mu_{n} R} .
$$

By substituting Eq. (5) in Eq. (3) we can write $S_{V}(f)$ as

$$
S_{V}(f)=\frac{q \mu_{n} R}{L^{2}} \frac{\alpha_{H}}{f^{\beta}} V^{2} .
$$

Fitting Eq. (6) with the experimental data yields $\beta=0.995$, very close to a perfect $1 / f$ dependence, and $\alpha_{H}=2.95 \times 10^{-4}$. 
TABLE I. Rectified dc voltage, NEP for $f>f_{C}$ and corner frequency, $f_{C}$, of the SSD array at different radiation powers.

\begin{tabular}{lccc}
\hline \hline Power $(\mathrm{dBm})$ & $V(\mathrm{mV})$ & $\mathrm{NEP}\left(\mathrm{pW} / \mathrm{Hz}^{1 / 2}\right)$ & $f_{C}(\mathrm{~Hz})$ \\
\hline-10 & 16 & 63.5 & $384 \mathrm{k}$ \\
-20 & 1.6 & 63.9 & $3.84 \mathrm{k}$ \\
-30 & 0.16 & 64.0 & 38.4 \\
\hline \hline
\end{tabular}

The specific microscopic mechanisms behind the observed $1 / f$ noise cannot be precisely determined, owning the large prediction variability of available theories. The energy fluctuation model ${ }^{18}$ with our material parameters would yield $\alpha_{H}=5 \times 10^{-3}$, whereas the prediction of Handel's theory ${ }^{19}$ is a few order of magnitude lower, $\alpha_{H}=10^{-5}-10^{-6}$. However, the measured $\alpha_{H}$ lies within the range observed for InGaAs 2DEGs, and it is consistent with mobility fluctuations due to electron interaction with phonons and material impurities as commonly reported. ${ }^{20-22}$ A more thorough discussion of $1 / f$ noise in SSD is out of the scope of this study. Equation (6) remains valid even when the device is used for detection at zero-bias. In this case, $V$ is the detected dc voltage which builds up across the rectifier.

It is worth noting that no generation-recombination noise ${ }^{16}$ with a typical Lorentzian spectrum, was observed. Generation-recombination noise might be expected from a device with a large surface-to-bulk ratio like the SSD, due to the interaction of electrons with the deep energy levels introduced by surface states. However, these energy states can be too deep to be observed at room temperature for the reported frequencies $>0.1 \mathrm{~Hz}$, and they might show a signature at higher temperature and/or lower frequencies.

The noise equivalent power (NEP), defined as the noise power density over the detection sensitivity, is a useful parameter to describe the noise performance of a detector. At frequencies high enough to neglect $1 / f$ excess noise, the NEP of the SSD array is simply proportional to the thermal noise and hence to the resistance of the whole array. Since connecting several rectifiers in parallel does not change the voltage sensitivity (the increased rectified current is loaded on a reduced resistance), the lower resistance immediately results in a lower NEP. The key feature of SSD arrays is the ability to connect a large number of SSDs in parallel without introducing parasitic elements such as interconnects, which would be extremely challenging in other competing technologies, e.g., Schottky diodes.

To discuss the impact of excess $1 / f$ noise, it is useful to consider the corner frequency $f_{C}$, i.e., the frequency at which the amount of $1 / \mathrm{f}$ noise equals the thermal noise. $f_{C}$ sets the magnitude of the lowest modulation frequency without considerable degradation of the NEP. As mentioned before, even with an unbiased rectifier the dc voltage that builds up on the terminals produces $1 / f$ noise, and $f_{C}$ will increase as higher radiation power are detected. Table I shows the NEP and $f_{C}$ of the unbiased SSD array at different radiation power levels. As shown, $f_{C}$ lies in a convenient frequency range $(\mathrm{kHz})$ for power below $-20 \mathrm{dBm}(10 \mu \mathrm{W})$, which is easily achievable with beam chopping and other modulation techni- ques. The measured NEP is comparable to those reported for zero-threshold Schottky diodes, but the $f_{C}$ is considerably lower for normal operation. ${ }^{13}$

In summary, we showed that SSD is a promising technology for microwave and $\mathrm{THz}$ detection. If used as a $\mathrm{THz}$ detector, the challenge is in the fabrication of antennas where a large number of SSDs can be connected in parallel. Viable structures such as interdigital antennas already exist and they have been demonstrated at $\mathrm{THz}$ frequencies. The only limit to the number of SSDs that can be placed in the gaps of the antenna fingers is the material cost and the availability of large-area fabrication techniques. Since the active area of SSD is defined by a single nanolithography step cost-effective, large-area lithography methods such as nanoimprinting (NIL) can be readily utilized. ${ }^{23}$ Moreover, multilevel NIL might enable the fabrication of the SSD array and the antenna in a single process step. $^{24,25}$

The authors wish to acknowledge the support of the EU FP7 project under Grant Agreement No. 243845 (ROOTHz) and UMIP.

${ }^{1}$ A. M. Song, M. Missous, P. Omling, A. R. Peaker, L. Samuelson, and W. Seifert, Appl. Phys. Lett. 83, 1881 (2003).

${ }^{2}$ C. Balocco, A. M. Song, M. Åberg, A. Forchel, T. González, J. Mateos, I. Maximov, M. Missous, A. A. Rezazadeh, J. Saijets, L. Samuelson, D. Wallin, K. Williams, L. Worschech, and H. Q. Xu, Nano Lett. 5, 1423 (2005).

${ }^{3}$ C. Balocco, S. R. Kasjoo, X. F. Lu, L. Q. Zhang, Y. Alimi, S. Winnerl, and A. M. Song, Appl. Phys. Lett. 98, 223501 (2011).

${ }^{4}$ C. Balocco, M. Halsall, N. Q. Vinh, and A. M. Song, J. Phys.: Condens. Matter 20, 384203 (2008)

${ }^{5}$ J. Mateos, B. G. Vasallo, D. Pardo, and T. González, Appl. Phys. Lett. 86, 212103 (2005).

${ }^{6}$ K. Y. Xu, G. Wang, and A. M. Song, Appl. Phys. Lett. 93, 233506 (2008).

${ }^{7}$ G. Farhi, E. Saracco, J. Beerens, D. Morris, S. A. Charlebois, and J.-P. Raskin, Solid-State Electron. 51, 1245 (2007).

${ }^{8}$ L. A. Majewski, C. Balocco, R. King, S. Whitelegg, and A. M. Song, Mater. Sci. Eng. B 147, 289 (2008).

${ }^{9}$ J. Kettle, R. M. Perks, and R. T. Hoyle, Electron. Lett. 45, 79 (2009).

${ }^{10}$ I. Iñiguez-de-la-Torre, J. Mateos, D. Pardo, A. M. Song, and T. González, Appl. Phys. Lett. 94, 093512 (2009).

${ }^{11}$ I. Iñiguez-de-la-Torre, J. Mateos, D. Pardo, and T. González, J. Appl. Phys. 103, 024502 (2008).

${ }^{12}$ M. Åberg, J. Saijets, A. M. Song, and M. Prunnila, Phys. Scr. T 114, 123 (2004).

${ }^{13}$ F. Sizov and A. Rogalski, Prog. Quant. Electron. 34, 278 (2010).

${ }^{14}$ M. Dyakonov and M. Shur, IEEE Trans. Electron Devices 43, 380 (1996).

${ }^{15}$ S. M. Sze, Semiconductor Devices, Physics and Technology, 2nd ed. (John Wiley \& Sons, Hoboken, NJ, 2002).

${ }^{16}$ A. Van der Ziel, Noise; Sources, Characterization, Measurement, (Prentice-Hall, Englewood Cliffs, NJ, 1970).

${ }^{17}$ A. Van der Ziel, Proc. IEEE 76, 233 (1988).

${ }^{18}$ T. Musha and M. Tacano, Physica A 346, 339 (2005).

${ }^{19}$ P. H. Handel, IEEE Trans. Electron. Devices 41, 2023 (1994).

${ }^{20}$ J. Pavelka, M. Tacano, N. Tanuma, and J. Šikula, Phys. Status Solidi C 8, 303 (2011).

${ }^{21}$ J. Berntgen, A. Behres, J. Kluth, K. Heime, W. Daumann, U. Auer, and F.-J. Tegude, Microelectron. Reliab. 40, 1911 (2000).

${ }^{22}$ M. E. Levinshtein, S. L. Rumyantsev, R. Tauk, S. Boubanga, N. Dyakonova, W. Knap, A. Shchepetov, S. Bollaert, and Y. Rollens, M. S. Shur, J. Appl. Phys. 102, 064506 (2007).

${ }^{23}$ J. Kettle, S. Whitelegg, A. M. Song, M. B. Madec, S. Yeates, M. L. Turner, L. Kotacka, and V. Kolarik, J. Vac. Sci. Technol. B 27, 2801 (2009).

${ }^{24}$ E. Lausecker, Y. Huang, T. Fromherz, J. C. Sturm, and S. Wagner, Appl. Phys. Lett. 96, 263501 (2010).

${ }^{25}$ Y. Huang, B. Hekmatshoar, S. Wagner, and J. C. Sturm, IEEE Electron Device Lett. 29, 737 (2008). 